P56 (continued)

Campbell, PhD, University of Oklahoma Health Sciences Center, Hudson College of Public Health; Beth DeGrace, $P h D$, University of Oklahoma Health Sciences Center, College of Allied Health; Jonathan Baldwin, MS, CNMT, RT (CT), University of Oklahoma Health Sciences Center, College of Allied Health

Background: One-fifth of children and adolescents have an abnormal serum cholesterol measure. Diets higher in plant-based foods and lower in sodium, sugar, cholesterol, and saturated fat reduce cardiovascular disease risk.

Objective: The purpose of this study is to determine if greater home access to fruits, vegetables, and snacks is associated with cardiac dietary heath at home and Early Care and Education (ECE) centers in preschool-age children and also to determine if there is a difference in children's cardiac dietary health between home and ECE.

Study Design, Settings, Participants: This cross-sectional study involved children (3-5 years old, $\mathrm{n}=88)$ and their primary caregivers from 15 licensed ECEs across Oklahoma. Parents reported home food access using the Healthy Home Survey and child's home dinner intake using the 3-Dinner Dietary Recall. Child ECE lunch consumption was recorded using the Dietary Observation for Child Care. The cardiac dietary score was composed of 6 variables, each with a single point to be summed: consumption of fish, fruits, vegetables, fiber, and limited sodium and sugary drinks.

Measurable Outcome/Analysis: Outcome variables were analyzed by using means, standard deviations, median, and frequencies. Adjusted linear regression analyses examined the relationship between home food access and cardiac dietary scores at home and ECE.

Results: At home, total fruit and vegetable (16.2 \pm 6.3$)$ outnumbered snacks $(5.5 \pm 3.0)$ with mean ratio of total home fruits and vegetables to snacks at $3.8 \pm 2.7$. There was no difference in cardiac dietary score between ECE $(1.50 \pm 0.8)$ and home $(1.27 \pm 0.9, P=0.0851)$. Children within both environments did not meet intake recommendations for most variables (vegetables [18.2-23.9\%], fruit [5.7-10.2\%], fish [4.5-10.2\%], fiber [1.1\%], sodium [21.6-38.6\%]). There was no relationship between home food access variables and cardiac dietary scores at home or ECE.

Conclusion: The cardiac dietary health of children at home and ECE does not meet recommendations. Interventions might consider the ECE setting to promote healthy eating among children and educate families on healthy eating at home.

Funding: None.

\section{P57 MOTHeRS' Project: Acceptability of a Medically Tailored Food Bag Treating Food Insecurity of High-Risk Pregnant Patients}

Kay Craven, MPH, LDN, RDN, CDCES, Brody School of Medicine at East Carolina University; Kathryn M Kolasa, PhD, LDN, RDN, kolasaka@ecu.edu, Brody School of
Medicine at East Carolina University, 101 Heart Dr, Greenville NC 27834; Brittany Smith, MS, East Carolina University, Department of Nutrition Science; Lauren Sastre, PhD, LDN, RDN, East Carolina University, Department of Nutrition Science

Objective: To develop a medically tailored emergency food bag and nutrition education handouts treating food insecurity (FIS) identified in clinical settings of rural, highrisk pregnant women.

Use of Theory: Grounded in the socio-ecological model Target Audience: High-risk pregnant women who screen positive for FIS during an appointment.

Program Description: The MOTHeRS' Project is a pilot to provide mental health and maternal-fetal services via telehealth to women with high-risk pregnancies in rural OB-GYN practices where FIS rates of $18-24 \%$ exceed the state average (15\%). The funder requested FIS be addressed but the timeline precluded us from interacting with the target audience or reviewing clinic records. An extensive literature review identified 9 nutrients critical but commonly under consumed in pregnancy. Published food lists from USDA and NIH were used to select foods high in the target nutrients. An online local grocery store was used to select 31 foods within the budget. Three complementary handouts were developed (eg, healthy eating, recipes, food safety) in English and Spanish. The bag and educational materials were designed to complement WIC benefits, expecting women may also receive WIC. The food bag and handouts were evaluated by 18 practitioners and/or researchers with expertise with rural, under-served pregnant women, and/or FIS. Bags were provided to patients who screened positive for FIS via the Hunger Vital Sign screener validated for clinical settings.

Evaluation Methods: Process evaluation was via a semistructured telephone interview with content validated for MOTHeRS'. Interviews were audio-recorded, transcribed verbatim and analyzed using deductive content analysis to identify themes. Transcripts were reviewed independently by the research team $(n=4)$ using a codebook and consensus was reached regarding themes.

Results: Preliminary themes suggest high satisfaction, acceptance and utilization of the food. Most participants reported limited access to other food resources and sharing food with other household members.

Conclusion: Our findings align with previous studies further suggesting medically tailored food resources provided in clinical settings are acceptable, and potentially associated with reduced social stigma. Contents of food bag and handouts will be shared.

Funding: United Health Foundation.

\section{P58 Online Test-Retest Reliability of the COAST Malnutrition Screening Tool}

Wendy Dahl,PhD, RD, wdahl@ufl.edu, University of Florida, 359 FSHN Building 572 Newell Dr, Gainesville, FL, 
P58 (continued)

32611; Alison O'Donoughue, MS, University of Florida; Karima Alabasi, PhD, University of Florida

Background: In face-to-face testing, the Comprehensive Older Adult Screening Tool (COAST) demonstrated internal consistency reliability and validity as a screening tool for malnutrition risk in community-dwelling older adults and its ease of use suggests it is a practical tool. However, the test-retest reliability of COAST has not been established.

Objective: The aim of this study was to determine the test-retest reliability of the COAST malnutrition screening tool in an online setting.

Study Design, Settings, Participants: Older adults (>60 years) were recruited through https://www.research match.org. Interested volunteers were emailed a Qualtrics link to the consent description and the 7-item COAST, which examines weight loss, decreased appetite, illness affecting food intake, diet quality, and intake of protein foods, at 2 timepoints, 2 weeks apart. The COAST was scored from 0 to 8 points, with 0-4 points denoting a high risk of malnutrition, 5-6 points indicating a moderate risk of malnutrition, and 7-8, a low risk of malnutrition.

Measurable Outcomes/Analysis: The primary outcome was the correlation of COAST scores at 2 timepoints. Results: Of the 947 sent the study description, 111 older adults agreed to be contacted by email. Of these, 49 completed the COAST at 2 timepoints. The COAST test-retest reliability coefficient was 0.74 , considered acceptable reliability for a malnutrition screening tool; $75 \%$ of participants were similarly categorized as high risk, moderate risk, or low risk of malnutrition at both timepoints. Inconsistent responses to the COAST question, "In general, how healthy is your overall diet?" explained 50\% of the misclassifications suggesting a need to clarify this item.

Conclusion: The COAST malnutrition screening tool demonstrated acceptable test-retest reliability when used in an online format. As the tool was previously validated for face-to-face administration, future research to determine the test-retest reliability using face-to-face administration may be warranted.

Funding: None.

\section{P59 Parenting and Snacks: A Mixed Methods Study to Guide Intervention Development}

VirginiaGray,PhD, RDN, virginia.gray@csulb.edu, California State University Long Beach, 1250 Bellflower Blvd, Long Beach, CA, 90840; Youngok Jung, PhD, California State University Long Beach; Jyotsna Pattnaik, EdD, California State University Long Beach; Nancy Dayne, EdD, California State University Long Beach; Sarah Domino, BS, California State University Long Beach; Haley Adel, BS, California State University Long Beach

Background: Recent work in family nutrition suggests a focus on snacking, since children's snacks are often lower in nutrients than foods served at mealtime and have increased in frequency and caloric contribution.
Developing healthy snacking habits in early life may impact the trajectory of snacking behaviors later in life.

Objective: To explore the relationships between snacking patterns and snack-related parenting; and drivers of snacking decisions and desired support for parents.

Study Design, Setting, Participants: A mixed-methods approach was used among student parents whose child(ren) attended a preschool program at a Southern California university.

Measurable Outcome/Analysis: A modified version of the Snack Intake Frequency Questionnaire and the Parenting around Snacking Questionnaire (P-SNAQ) were used to explore correlations between snacking patterns and snack-related parenting. Three focus groups were conducted to identify drivers of snacking decisions and to guide design of healthy snacking intervention strategies. Focus group transcripts were coded using thematic analysis by 2 independent coders.

Results: Survey participants $(n=42)$ were predominantly female $(n=35)$ and identified as Hispanic/Latino $(n=19)$. Findings indicated positive correlations between frequency of offering healthy snacks and reasoning/support for healthy snacks $(\mathrm{r}=0.43, P=0.006)$ and snack planning/routines $(\mathrm{r}=0.37, P=0.02)$; a negative correlation between frequency of unhealthy snacks and availability of healthy snacks ( $\mathrm{r}=-0.32, P=0.04)$; and negative correlations between parent age and emotion-based snacking $(\mathrm{r}=-0.46$, $P=0.003)$, snacks as rewards $(\mathrm{r}=-0.35, P=0.03)$, and snacks to manage behavior $(\mathrm{r}=-0.45, P=0.005)$. Focus group findings $(\mathrm{n}=11)$ suggest parents balance priorities when making snacking decisions, including health, behavior control, convenience/accessibility, parent/child roles, and social influences. Parents suggested a snacking-focused class with information on snacking strategies and social support.

Conclusion: Findings suggest younger parents may benefit from snacking-focused intervention programs that support healthy snacking routines and alternative behavior management strategies for children.

Funding: California State University Long Beach Office of Research and Sponsored Programs.

\section{P60 Parents Perceptions of Their Personal Eating Habits and Their Child's Eating Behaviors}

Angelique C. Munoz, MS, LD, RDN, Harris Health System; Vicky L. Green, PhD, LDN, RDN, vgreen@latech.edu, Louisiana Tech University, PO Box 3167, Ruston, LA, 71272; Simone P. Camel, PhD, LD, RDN, Louisiana Tech University; Mary C. Fontenot, $P h D, L D N, R D N$, Louisiana Tech University

Background: Parent perception of their child's and their own eating habits may influence feeding and eating behaviors.

Objective: To evaluate associations between the parent's perceptions of their own eating habits and their perception of their child's eating habits.

Study Design, Setting, Participants: A cross-sec-

Continued on page 552 